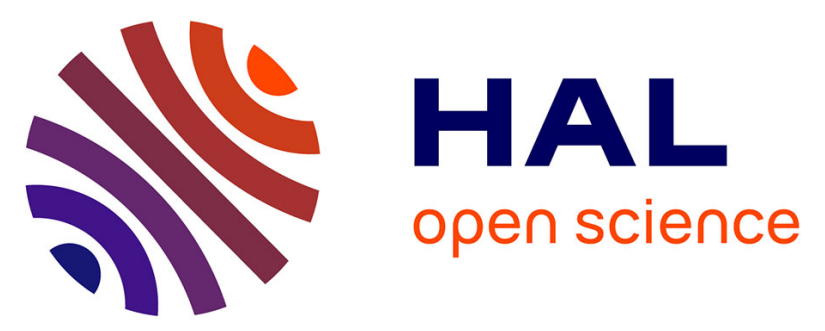

\title{
Calculation of total cross sections and effective emission coefficients for B5 + collisions with ground-state and excited hydrogen
}

\author{
F Guzmán, L F Errea, Clara Illescas, L Méndez, B Pons
}

\section{- To cite this version:}

F Guzmán, L F Errea, Clara Illescas, L Méndez, B Pons. Calculation of total cross sections and effective emission coefficients for B5 + collisions with ground-state and excited hydrogen. Journal of Physics B: Atomic, Molecular and Optical Physics, 2010, 43 (14), pp.144007. 10.1088/09534075/43/14/144007. hal-00597860

\section{HAL Id: hal-00597860 \\ https://hal.science/hal-00597860}

Submitted on 2 Jun 2011

HAL is a multi-disciplinary open access archive for the deposit and dissemination of scientific research documents, whether they are published or not. The documents may come from teaching and research institutions in France or abroad, or from public or private research centers.
L'archive ouverte pluridisciplinaire HAL, est destinée au dépôt et à la diffusion de documents scientifiques de niveau recherche, publiés ou non, émanant des établissements d'enseignement et de recherche français ou étrangers, des laboratoires publics ou privés. 


\title{
Calculation of total cross sections and effective emission coefficients for $\mathrm{B}^{5+}$ collisions with ground-state and excited hydrogen.
}

\author{
F. Guzmán ${ }^{\dagger}$, L. F. Errea ${ }^{\ddagger}$, Clara Illescas $^{\ddagger}$, L. Méndez Mend B. $^{\ddagger}$ and \\ Pons $\S$ \\ $\dagger$ ADAS-EU. Department of Physics. University of Strathclyde, Glasgow. Present \\ address: office 125, Building 9.1 IEF-4, Forschungszentrum Juelich, 52425 Juelich, \\ Germany. \\ ${ }^{\ddagger}$ Laboratorio Asociado al CIEMAT de Física Atómica y Molecular en Plasmas de \\ Fusión. Departamento de Química, Universidad Autónoma de Madrid, \\ Madrid-28049, Spain \\ $\S$ Centre de Lasers Intenses et Applications. Université de Bordeaux-I-CNRS-CEA. \\ 351 Cours de la Liberation 33405, Talence, France.
}

\begin{abstract}
Classical and semiclassical calculations of $n l$-resolved charge exchange cross sections in $\mathrm{B}^{5+}$ collisions with $\mathrm{H}\left(n_{i}\right)$ are performed to compute effective emission coefficients for the $n=7 \rightarrow n=6$ transition in $\mathrm{B}^{4+}$ for plasma conditions typical of the ASDEX-U tokamak. For $n_{i}=1$, the value of the emission coefficient is larger than that obtained from ADAS database by a factor of two at energies of $10 \mathrm{keV} / \mathrm{amu}$, but no differences are found at energies above $50 \mathrm{keV} / \mathrm{amu}$. For $n_{i}=2$, our calculation yields emission coefficients close to those derived from ADAS data from low to high impact energies. The emission coefficients corresponding to $\mathrm{B}^{5+}+\mathrm{H}\left(n_{i}=3\right)$ collisions are of the same order of magnitude than those for $n_{i}=2$.
\end{abstract}

PACS numbers: $34.70 .+\mathrm{e}, 34.10 .+\mathrm{x}$ 


\section{Introduction}

Information on plasma core characteristics is currently obtained by employing diagnostic beams, and these techniques will be also the main diagnostic tool of ITER ([1] and references therein). In particular, in charge exchange recombination spectroscopy (CXRS) (see, e.g., [2]), neutral beams are injected in the plasma, and charge exchange (CX) occurs in collisions with plasma impurity ions. Although other atoms ( $\mathrm{He}, \mathrm{Li})$ have been employed, we shall focus on the application of the diagnostics based on $\mathrm{H}$ (or D) beams. For this particular case, the basic process is:

$$
\mathrm{H}+\mathrm{A}^{\mathrm{q}+} \rightarrow \mathrm{H}^{+}+\mathrm{A}^{(\mathrm{q}-1)+}
$$

The ion $\mathrm{A}^{(\mathrm{q}-1)+}$ is formed in an excited state and the ensuing emission is employed to determine plasma density and temperature. For energetic beams, the CX reaction occurs in the plasma center, where the impurities ions are fully stripped, and therefore the basic process is a one-electron reaction.

The application of CXRS requires the knowledge of high-precision state-selective cross sections for populating excited states $\mathrm{A}^{(\mathrm{q}-1)+}(n, l)$. These data are included in a collisional-radiative model that leads to the effective emission coefficients, which are afterwards applied in the diagnostic. The beam diagnostic techniques are therefore based on a set of atomic data, and those included in the Atomic Data and Analysis Structure (ADAS) database $\ddagger$ are often employed.

In previous works, we have calculated cross sections for several charge exchange reactions $[3,4,5]$ in ion collisions with $\mathrm{H}(1 \mathrm{~s})$. However, a small fraction of excited atoms $\mathrm{H}\left(n_{i}=2\right)$ can collide with the impurity ions; they are produced by interaction of ground state beam atoms with the plasma constituents. The main relevance of these excited atoms is the well-known fact that the CX cross section is in general larger than that for the corresponding process with ground state donors. In this respect, Hoekstra et al. [6] compared effective emission coefficients for collisions of several light ions with $\mathrm{H}\left(n_{i}=1\right)$ and $\mathrm{H}\left(n_{i}=2\right)$, and an example of the influence of excited donors on the measurements of impurity densities can be found in ref. [7]. Moreover, the work of ref. [8], on the CXRS diagnostics of $\mathrm{B}^{5+}$ density in the Alcator C-Mod tokamak, has pointed out that the lack of $n_{i}=2$ in the calculated effective emission coefficient leads to a misleading result that shows a spurious dependence on the beam energy. Also the lack of $n_{i}=3$ yields errors of $15 \%$ in the measured densities.

The importance of Boron ions lies on the fact that using B to coat the first wall (boronisation) improves the energy confinement [9]. In the present work, we present effective emission coefficients for the reactions:

$$
\begin{aligned}
& \mathrm{B}^{5+}+\mathrm{H}(1 \mathrm{~s}) \rightarrow \mathrm{B}^{4+}(n l)+\mathrm{H}^{+} \\
& \mathrm{B}^{5+}+\mathrm{H}\left(n_{i}=2,3\right) \rightarrow \mathrm{B}^{4+}(n l)+\mathrm{H}^{+}
\end{aligned}
$$

The cross sections for reaction (2) were calculated in [5]. Preliminary calculations for collisions with $\mathrm{H}(2 \mathrm{~s})$ were reported in [10]. In the present work we present new $\ddagger$ http://adas.phys.strath.ac.uk 
partial cross sections for reactions (3); they have been calculated using the classical and semiclassical formalisms as explained in [3]. At high collision energies ( $E>4 \mathrm{keV} / \mathrm{amu})$, we employ the Classical Trajectory Monte Carlo Method (CTMC). At low energies we have applied a semiclassical molecular method, and in particular, calculations with excited Hydrogen have been carried out as explained in [11]. Although the semiclassical results are relatively less important than the CTMC ones in view of their application in CXRS, since experiments take place at higher energies to those where the method is applicable, the comparison between CTMC and semiclassical results allows us to discuss the accuracy of our classical calculation. Besides we shall compare our results with the cross sections reported in ref. [6] which employed a CTMC method, and with those available in ADAS database, reported by the authors of ref. [6]. Classical calculations for collisions with $\mathrm{H}\left(n_{i}=3\right)$ have been also carried out because of the potential relevance of these reactions in diagnostics [8]. In this respect it must be noted that cross sections and effective emission coefficients employed in ref. [8] for $n_{i}=3$ donors were obtained by scaling the CX cross sections from $\mathrm{H}\left(n_{i}=1,2\right)$.

At the collision energies typical of diagnostic beams (above $25 \mathrm{keV} / \mathrm{amu}$ ), ionization is the dominant process. In the present work we have also evaluated, using the CTMC method, the total cross section for the reaction:

$$
\mathrm{B}^{5+}+\mathrm{H}\left(n_{i}=2,3\right) \rightarrow \mathrm{B}^{5+}+\mathrm{H}^{+}+\mathrm{e}^{-},
$$

which is relevant to calculate beam stopping coefficients.

Atomic units are employed unless otherwise stated.

\section{Theory}

\subsection{Eikonal-CTMC formalism.}

The CTMC treatment has been explained in previous publications and we shall only summarize it. First we assume (eikonal approximation) that the nuclei follow rectilinear trajectories with constant velocity $\boldsymbol{v}$ and impact parameter $\boldsymbol{b}(\boldsymbol{R}=\boldsymbol{v} t+\boldsymbol{b})$. It is known that this approximation is practically exact for collision energies above $100 \mathrm{eV}$. The electron dynamics is described by the statistical phase space distribution which satisfies the classical Liouville equation:

$$
\frac{\partial \rho(\boldsymbol{r}, \boldsymbol{p}, t ; v, b)}{\partial t}=-[\rho(\boldsymbol{r}, \boldsymbol{p}, t ; v, b), H]
$$

where $H$ is the classical Hamiltonian for fixed nuclei:

$$
H=\frac{p^{2}}{2}-\frac{Z_{\mathrm{A}}}{r_{\mathrm{B}}}-\frac{Z_{\mathrm{H}}}{r_{\mathrm{H}}}
$$

with $Z_{\mathrm{B}, \mathrm{H}}$ the nuclear charges and $r_{\mathrm{B}, \mathrm{H}}$ the electron distances to both nuclei. In the CTMC method the electron distribution is discretized using $\mathcal{N}$ classical trajectories [12]:

$$
\rho(\boldsymbol{r}, \boldsymbol{p}, t ; v, b)=\frac{1}{\mathcal{N}} \sum_{j=1}^{\mathcal{N}} \delta\left(\boldsymbol{r}-\boldsymbol{r}_{j}(t)\right) \delta\left(\boldsymbol{p}-\boldsymbol{p}_{j}(t)\right)
$$


and substitution of eq. (7) in eq. (5) yields the Hamilton equations for the individual trajectories $\boldsymbol{r}_{j}(t), \boldsymbol{p}_{j}(t)$ :

$$
\dot{\boldsymbol{r}_{j}}=\frac{\partial H}{\partial \boldsymbol{p}_{j}} ; \dot{\boldsymbol{p}_{j}}=-\frac{\partial H}{\partial \boldsymbol{r}_{\boldsymbol{j}}}
$$

In general, the accuracy of capture and ionization cross sections basically depends on that of the initial distribution. In previous works we have employed either the microcanonical or the hydrogenic distributions. In the microcanonical one [12], all electronic trajectories have the energy of the initial quantum state $\epsilon=\epsilon_{0}$. The hydrogenic distribution [13] is obtained by means of a linear combination of $N$ microcanonical distributions with an average energy $\langle\epsilon\rangle=\epsilon_{0}$ (e.g. [3]). For excited atomic states $\left(\mathrm{H}\left(n_{i}=2\right)\right)$ a better description is achieved, as shown in figure 1 , by using an initial gaussian distribution [14]:

$$
\rho(\boldsymbol{r}, \boldsymbol{p})=2^{-1 / 2} \pi^{-3} \int(-\epsilon)^{5 / 2} \rho(\epsilon) \delta(H-\epsilon) \mathrm{d} \epsilon
$$

with

$$
\rho(\epsilon)=K_{1} \mathrm{e}^{-K_{2}\left(n_{\mathrm{H}}-2.2\right)^{2}} \quad ; \quad n_{\mathrm{H}}(\epsilon)=Z_{\mathrm{H}} / \sqrt{-2 \epsilon}
$$

and where the constants $K_{1}$ and $K_{2}$ are determined by the conditions of normalization and mean energy $\left\langle\epsilon>=\epsilon_{0}\right.$. In practice, the integral in (9) has been evaluated numerically by including 60 values of $\epsilon$ in the interval $-0.243<\epsilon \leq-0.08336$; these limits are those given by the criterion of Becker and McKellar [15], which defines the box in the phase space that corresponds to the $n=2$ quantum level. In general, the box for the quantum level $n$ is:

$$
n(n-1 / 2)(n-1) \leq n_{\mathrm{H}}^{3} \leq n(n+1 / 2)(n+1)
$$

where $n_{\mathrm{H}}$ is the classical quantum number defined in eq. (10).

The initial distributions for $\mathrm{H}(2 \mathrm{~s})$ and $\mathrm{H}(2 \mathrm{p})$ have been obtained by retaining only those trajectories with angular momentum $l_{c}$ fulfilling $0<n l_{c} / n_{\mathrm{H}}<1$ and $1<n l_{c} / n_{\mathrm{H}}<2$, respectively, with $n=2$ (see [15]). To illustrate the comparison of the classical distributions we plot in Fig. 1 the values of the radial components of coordinate and momentum distributions:

$$
\begin{aligned}
& \rho^{C}(r)=\int \mathrm{d} \boldsymbol{p} \int_{0}^{2 \pi} \mathrm{d} \phi \int_{0}^{\pi} \mathrm{d} \theta \sin \theta r^{2} \rho_{2 s, 2 p}(\boldsymbol{r}, \boldsymbol{p}) \\
& \rho^{C}(p)=\int \mathrm{d} \boldsymbol{r} \int_{0}^{2 \pi} \mathrm{d} \phi_{p} \int_{0}^{\pi} \mathrm{d} \theta_{p} \sin \theta_{p} p^{2} \rho_{2 s, 2 p}(\boldsymbol{r}, \boldsymbol{p})
\end{aligned}
$$

and the corresponding quantities for the quantum mechanical distributions:

$$
\begin{aligned}
& \rho^{Q M}(r)=\int_{0}^{2 \pi} \mathrm{d} \phi \int_{0}^{\pi} \mathrm{d} \theta \sin \theta r^{2}\left|\Phi_{2 s, 2 p}(\boldsymbol{r})\right|^{2} \\
& \rho^{Q M}(p)=\int_{0}^{2 \pi} \mathrm{d} \phi_{p} \int_{0}^{\pi} \theta_{p} \sin \theta_{p} p^{2}\left|\bar{\Phi}_{2 s, 2 p}(\boldsymbol{p})\right|^{2}
\end{aligned}
$$



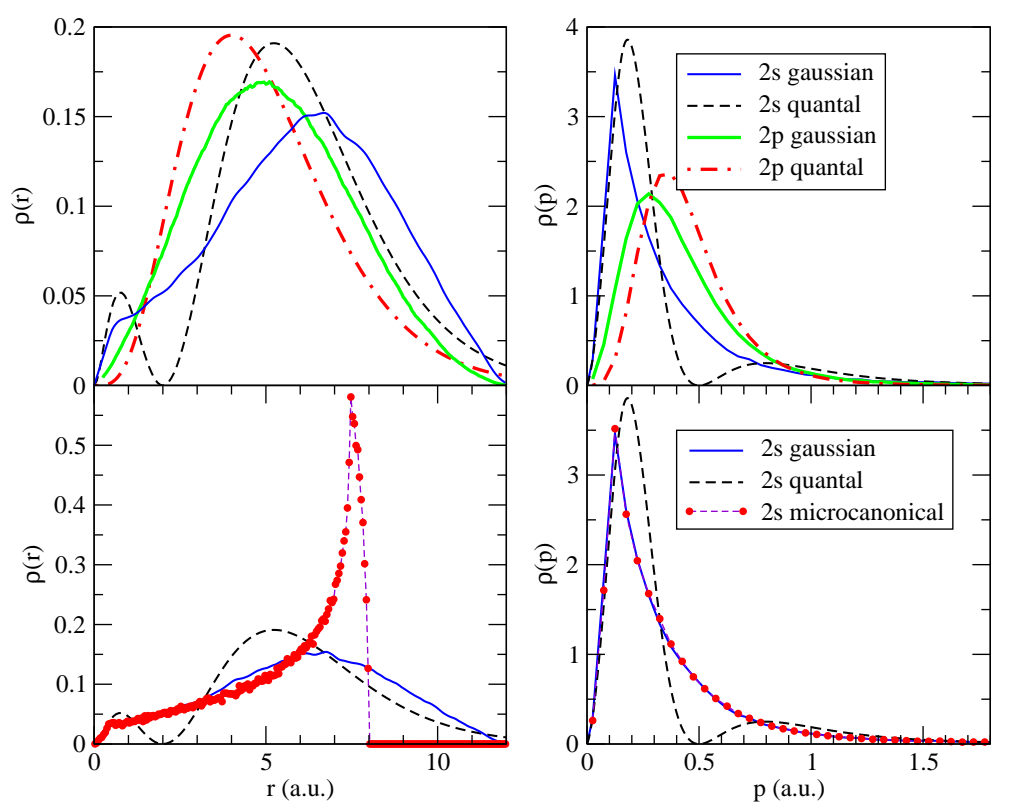

Figure 1. Comparison between the exact quantum mechanical initial distributions for $\mathrm{H}(2 \mathrm{~s})$ and $\mathrm{H}(2 \mathrm{p})$ and the corresponding gaussian distributions (eqs. (13), (15). For comparison purposes the microcanonical distribution for $\mathrm{H}(2 \mathrm{~s})$ is also shown.

It is important to note that, as for the case of $\mathrm{H}(1 \mathrm{~s})$ [14], the use of the gaussian distribution does not appreciably modify the momentum distribution, but notably improves the coordinate distribution with respect to the microcanonical one.

In the calculation we have employed a set of $2 \times 10^{5}$ electron trajectories and the Hamilton equations were solved up to $t=t_{\max }=150$. We apply an energy criterion to evaluate ionization, capture and excitation cross sections, by considering the electron energies with respect to both $(\mathrm{H}$ and $\mathrm{B})$ moving nuclei. Namely, trajectories leading to ionization fulfill (the origin of the electronic coordinates is placed on the $\mathrm{H}$ nucleus):

$$
\epsilon_{\mathrm{H}}=p^{2} / 2-1 / r>0 ; \epsilon_{\mathrm{B}}=1 / 2(\boldsymbol{p}-\boldsymbol{v})^{2}-Z_{B} /\left|\boldsymbol{r}-\boldsymbol{b}-\boldsymbol{v} t_{\max }\right|>0
$$

while for capture we have: $\epsilon_{\mathrm{H}}>0, \epsilon_{\mathrm{B}}<0$, and for excitation: $\epsilon_{\mathrm{H}}<0, \epsilon_{\mathrm{B}}>0$. The probability for a process $\mathrm{X}$ reads:

$$
P_{\mathrm{X}}(v, b)=\int \mathrm{d} \boldsymbol{r} \int \mathrm{d} \boldsymbol{p} \rho_{\mathrm{X}}\left(\boldsymbol{r}, \boldsymbol{p}, t_{\max } v, b\right)=\mathcal{N}_{\mathrm{X}} / \mathcal{N}
$$

where $\mathcal{N}_{\mathrm{X}}$ is the number of trajectories that lead to process X. Partial $n l$ cross sections were obtained using the criterion of ref. [15]. The value of $n$ associated to a given trajectory is obtained by applying the box definition of eq. (11) for the $\mathrm{B}^{4+}$ ion; i.e., $n$ fulfills $n(n-1 / 2)(n-1) \leq n_{\mathrm{B}}^{3} \leq n(n+1 / 2)(n+1)$ with $n_{\mathrm{B}}=Z_{B} / \sqrt{-2 \epsilon_{B}}$. Similarly, the value of $l$ is obtained from the condition:

$$
l \leq n l_{c} / n_{\mathrm{B}} \leq l+1
$$




\subsection{Semi-classical molecular expansion.}

As in the CTMC treatment the nuclei follow straight-line trajectories while the electronic motion is described quantum-mechanically by means of the wavefunction $\Psi$, solution of the equation:

$$
\left[\widehat{H}-\left.\mathrm{i} \frac{\partial}{\partial t}\right|_{\boldsymbol{r}}\right] \Psi(\boldsymbol{r}, t ; v, b)=0 \quad ; \quad \widehat{H}=-\frac{1}{2} \nabla^{2}-\frac{Z_{\mathrm{B}}}{r_{\mathrm{B}}}-\frac{Z_{\mathrm{H}}}{r_{\mathrm{H}}}
$$

The collision wavefunction $\Psi$ is expanded as a linear combination of molecular orbitals (OEDMs, for One Electron Diatomic Molecules) $\left\{\chi_{k}\right\}$ in the form:

$$
\Psi(\boldsymbol{r}, t ; v, b)=e^{\mathrm{i} U(\boldsymbol{r}, t)} \sum_{k} a_{k}(t ; v, b) \chi_{k}(\boldsymbol{r}, R) e^{-\mathrm{i} \int_{0}^{t} \varepsilon_{k}\left(t^{\prime}\right) d t^{\prime}}
$$

where the OEDMS are eigenfunctions of $\widehat{H}$ with energies $\varepsilon_{k}(R)$ and $U$ is a common translation factor [16], defined in terms of the switching function proposed in ref. [17].

The molecular basis set includes 223 OEDMs, which are: the entrance channels of reactions (2) and (3), which dissociate into $\mathrm{B}^{5+}+\mathrm{H}(n=1,2)$, and the OEDMs dissociating into $\mathrm{B}^{4+}(n l)+\mathrm{H}(1 \mathrm{~s})$ with $1 \leq n \leq 10$. The correlation diagram of the $\sigma$ molecular orbitals is shown in fig. 2, where we plot the effective quantum numbers $n_{\text {eff }}^{k}=6 / \sqrt{-2 \varepsilon_{k}}$ as functions of the internuclear distance $R$. The cross sections for CX and ionization from $\mathrm{H}\left(n_{i}=2\right), \sigma\left(n_{i}=2\right)$ is obtained as:

$$
\sigma\left(n_{i}=2\right)=\frac{1}{4}\left(\sigma(2 \mathrm{~s})+\sigma\left(2 \mathrm{p}_{\mathrm{z}}\right)+\sigma\left(2 \mathrm{p}_{\mathrm{x}}\right)+\sigma\left(2 \mathrm{p}_{\mathrm{y}}\right)\right)
$$

where the cross sections in the right hand side are obtained in the calculation where the initial states are the corresponding atomic orbitals defined in the rotating molecular frame, with $\hat{Z} \| \hat{R}$ and $\hat{Y}$ perpendicular to the collision plane. The OEDMS 980 and 860 (labelled according to their united-atom limit) correlate as $R \rightarrow \infty$ to the linear combinations $\sqrt{2}\left(2 s \pm 2 p_{z}\right)$, and the atomic orbitals are therefore obtained by combining these molecular orbitals. Accordingly, the initial amplitudes of the molecular orbitals are given by [18]:

$$
\begin{aligned}
& a_{k}\left(t=-t_{\max }\right)=\exp \left[-\mathrm{i}\left(f_{k}-\int_{0}^{-t_{\max }} \varepsilon_{k} \mathrm{~d} t^{\prime}+\int_{0}^{-\infty} E_{N_{0} L_{0} M_{0}} \mathrm{~d} t^{\prime}\right)\right] \\
& \times\left\langle\chi_{k} \mid \Phi_{N_{0} L_{0} M_{0}}\right\rangle
\end{aligned}
$$

where, in $\mathrm{B}^{5+}+\mathrm{H}(2 \mathrm{~s}, 2 \mathrm{p})$ collisions:

$$
f_{k}=\frac{Z_{\mathrm{B}} d}{b v} \tan ^{-1} \frac{b}{v t_{\max }} .
$$

$d=3 \mathrm{a}_{0}$ is the $2 \mathrm{~s}-2 \mathrm{p}$ transition dipole moment and $N_{0}, L_{0}, M_{0}$ are the atomic quantum numbers (200 and 210 in the present case).

The contributions $\sigma\left(2 p_{x}\right)$ and $\sigma\left(2 p_{y}\right)$ are both evaluated by solving the system of differential equations with the system initially described by the OEDM 871. In the former case $\pi$ OEDMs are coupled with $\sigma$ and $\delta$ orbitals through rotational couplings, while in the latter case, where the system is initially described by a $\pi$ molecular orbital, perpendicular to the collision plane, all non-adiabatic couplings with $\sigma$ orbitals vanish. 


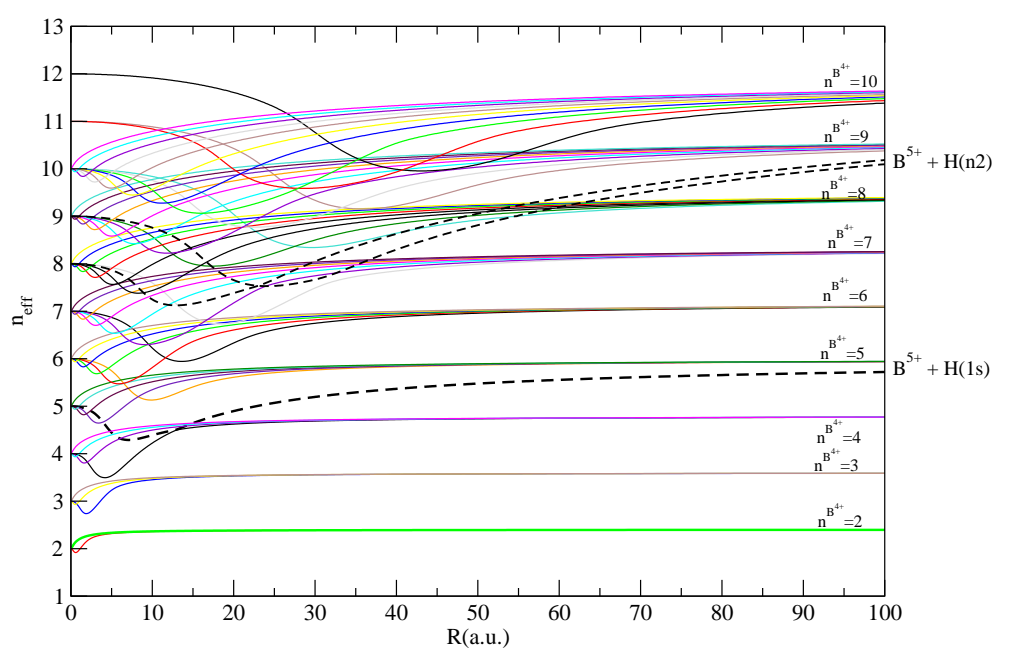

Figure 2. Effective quantum numbers $n_{\mathrm{eff}}^{k}=6 / \sqrt{-2 E_{k}}$ as functions of the internuclear distance $R$ for the OEDM of the $\mathrm{BH}^{5+}$ quasimolecule.

Substitution of equation (20) in (19) leads to a set of first-order differential equations for the expansion coefficients $a_{k}(t ; v, b)$. CX and excitation cross sections are obtained by integrating the corresponding probabilities over the impact parameter:

$$
\sigma_{n l m}^{B, H}(v)=2 \pi \int\left|a_{n l m}^{\mathrm{B}, \mathrm{H}}(t \rightarrow \infty ; v, b)\right|^{2} b \mathrm{~d} b=2 \pi \int P_{n l m}^{\mathrm{B}, \mathrm{H}}(v, b) b \mathrm{~d} b
$$

where the superscripts $\mathrm{B}, \mathrm{H}$ indicate that the electron is asymptotically bound to the $\mathrm{B}$ nucleus (charge exchange) or $\mathrm{H}$ (excitation). The coefficients $a_{n l m}$ are obtained by applying the extrapolation procedure proposed in [18] and accounting for the Stark effect and the residual rotation of the internuclear axis from $t=t_{\max }$ to $t=\infty$.

\section{Results and discussion}

\subsection{Cross sections}

The spectral lines usually employed in the CXRS diagnostics are those lying in the visible region of the spectrum. For the particular case of $\mathrm{B}(\mathrm{V})$ the diagnostic is normally based on the intensity of the $n=7 \rightarrow n=6$ line with $\lambda=494.47 \mathrm{~nm}$, and therefore the most important partial cross section is that for populating $\mathrm{B}^{4+}(n=7)$, which is shown in fig. 3. The main limitation of the OEDM treatment is the overpopulation of the excited orbitals due to the ionization flux that remains trapped in the CX channels; this can be clearly noted in the shape of the CX cross section in fig. 3. Therefore the connection between classical and quantal partial CX cross sections must be carried out at energies where the ionization cross section is small compared to the capture cross sections. For instance, in the case of population of $\mathrm{B}^{4+}(n=7)$, the semiclassical data cannot be employed for energies larger than $E \simeq 6 \mathrm{keV}$. Although the semiclassical results are not very significant in view of their application in CXRS, since experiments take place at higher energies to those where the method is applicable, they support the 
CTMC results and, in particular the initial distribution employed. In our calculation, the difference between the CX cross section from CTMC and OEDM results of fig. 3 is smaller than $5 \%$ at $E=4 \mathrm{keV} / \mathrm{amu}$.

In order to obtain a smooth joining of both calculations, different energy ranges have been employed for different $n$. Similarly, recommended $n l$-partial cross sections have been built by joining the classical and semiclassical branching ratios $r_{n l}=\frac{\sigma_{n l}}{\sigma_{n}}$ at the energy that yields the smoothest joining. As a check of the procedure, we have found that $\left|1-\sum_{l} r_{n l}\right|<0.05$. Our results are illustrated in fig. 4 for $\mathrm{B}^{5+}+\mathrm{H}(2 p) \rightarrow$ $\mathrm{B}^{4+}(n l)+\mathrm{H}^{+}$and $\mathrm{B}^{5+}+\mathrm{H}(n=2) \rightarrow \mathrm{B}^{4+}(n l)+\mathrm{H}^{+} \mathrm{CX}$ collisions with $n=6,7,8$. The $\sigma(2 p)$ and $\sigma(n=2)$ cross sections have respectively been computed according to the statistical averages $\left(\sigma\left(2 p_{x}\right)+\sigma\left(2 p_{y}\right)+\sigma\left(2 p_{z}\right)\right) / 3$ and $(3 \sigma(2 p)+\sigma(2 s)) / 4$ (see eq. $\left.(21)\right)$. Since $\sigma(2 s) \approx \sigma(2 p) / 3$, we find in Fig. 4 that $\sigma(n=2) \approx \sigma(2 p)$ for both $n$ - and $n l$ partial cross sections. One can further note in Fig. 4 that classical and semiclassical $n$-partial cross sections are smoothly joined, but the results are less smooth for the $n l$ partial cross sections corresponding to the lowest $l$ values $(l=0,1,2)$, although these cross sections are very small with respect to those associated to high $l$ values.

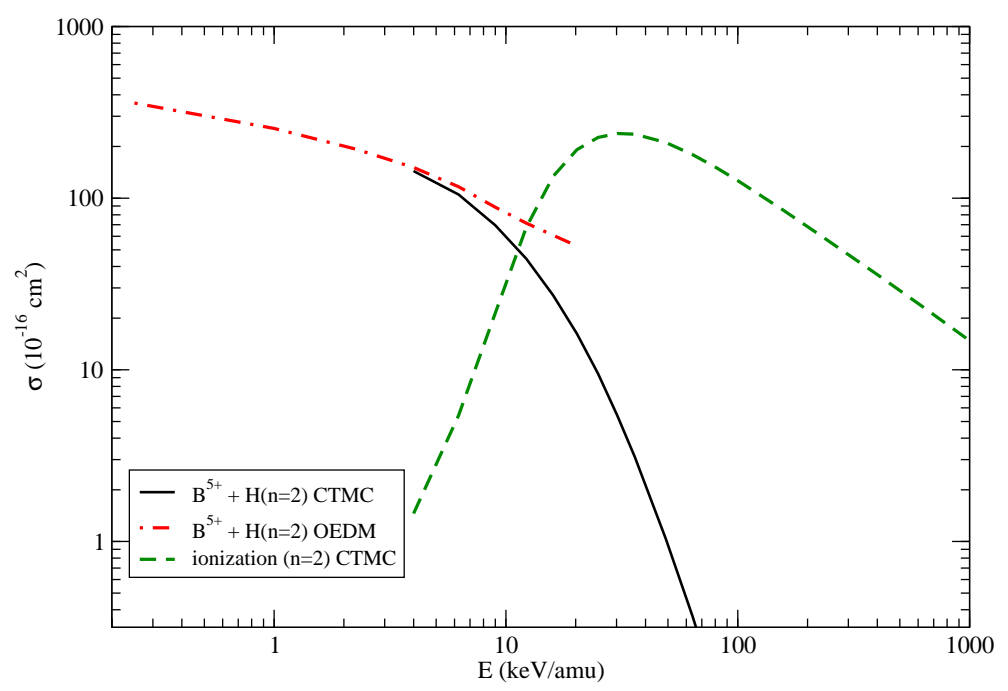

Figure 3. Total cross sections for CX into $\mathrm{Be}^{4+}(n=7)$ and ionization in collisions of $\mathrm{B}^{5+}$ with $\mathrm{H}(\mathrm{n}=2)$, calculated using CTMC and OEDM treatments.

Our recommended $n$-partial CX cross sections are shown in fig. 5, together with our results using a single microcanonical distribution, and the data included in ADAS. Our results are completely different from those of [6] (not shown in the figure). However the values stored in ADAS, which were obtained by the authors of ref. [6], agree with our values, which points to a mistake in the figure of that work. The comparison shows that total CX cross section for $\mathrm{B}^{5+}$ collisions with $\mathrm{H}\left(n_{i}=2\right)$ is accurately evaluated down to 

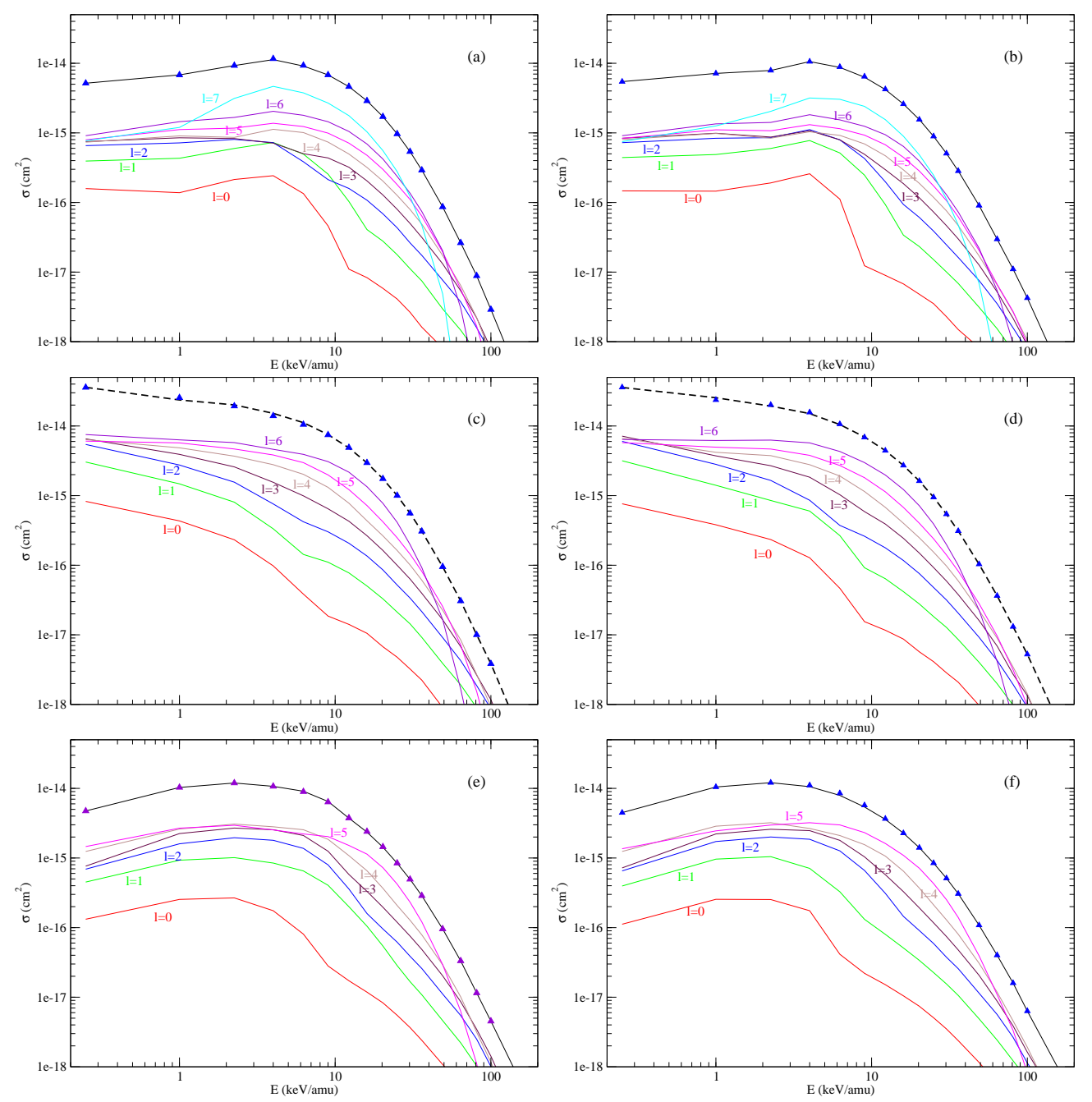

Figure 4. Partial cross sections for populating $\mathrm{B}^{4+}(n l)$ sublevels with $n=8$ (panels (a) and (b)), $n=7$ (panels (c) and (d)) and $n=6$ (panels (e) and (f)), in collisions of $\mathrm{B}^{5+}$ with $\mathrm{H}(2 p)$ (panels (a), (c) and (e)) and $\mathrm{H}(n=2)$ (panels (b), (d) and (f)). The highest line on each panel is the corresponding $\sigma_{n}$ cross section obtained by adding the corresponding $\sigma_{n l}$ while the triangles are the values obtained by joining the classical and semiclassical values for $\sigma_{n}$.

$E \approx 2 \mathrm{keV} / \mathrm{amu}$ by employing an initial microcanonical distribution. Such an accurate behaviour of microcanonical calculations at low $E$ is at first sight surprising, since previous CTMC calculations have shown that the initial condition has to be improved beyond the microcanonical framework to yield reliable cross sections (see, for instance, [3]). Nonetheless, most of these previous works have considered the ground-state $\mathrm{H}$ target, where it is necessary to improve the initial classical distribution by including a wide energy spread in the initial classical state in order to mimic underbarrier CX transitions from the entry channel [14]. The consequence of this procedure is that 


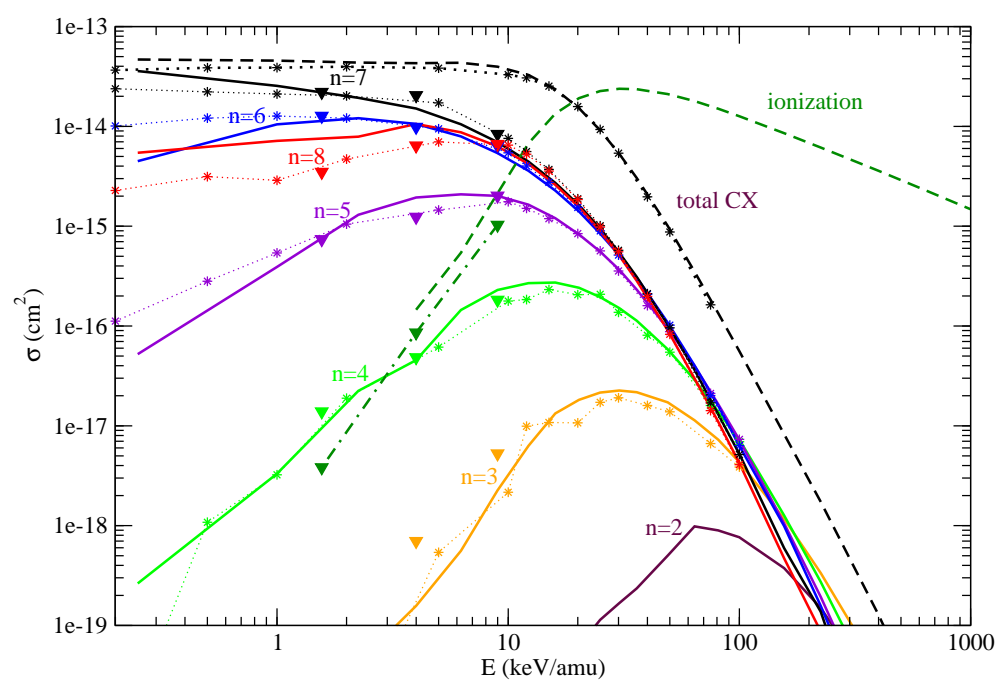

Figure 5. Total cross sections for $\mathrm{CX}$ into $\mathrm{Be}^{4+}(n)$ and ionization in collisions of $\mathrm{B}^{5+}$ with $\mathrm{H}(n=2)$. The lines are the recommended data obtained joining classical and semiclassical calculations. The triangles are the results of a classical calculation with a microcanonical initial distribution and the asterisks correspond to the data stored in ADAS, which are issued from the microcanonical CTMC calculations of [6].

the new distribution includes unphysically low energies, which lead to an unaccurate description of transitions to low $n$ levels. In the present calculation for collisions with $\mathrm{H}\left(n_{i}=2\right)$, although the initial microcanonical distribution does not correctly reproduce the tail of the quantal spatial distribution (see fig. 1), it agrees with the quantal distribution for $r \leq 7$, which explains the agreement of the total cross section. However, the corresponding partial cross sections for populating $\mathrm{B}^{4+}(n=6,7,8)$ of fig. 5 show noticeable differences with the recommended data.

Since Bespamyatnov et al. [8] have pointed out that excited donors $\mathrm{H}(n=3)$ can significantly influence the determination of the impurity density, we have performed a CTMC calculation of $\mathrm{CX}$ and ionization cross sections in $\mathrm{B}^{5+}+\mathrm{H}(n=3)$ collisions in the energy range $3 \leq E \leq 1000 \mathrm{keV} / \mathrm{amu}$. We have employed the microcanonical distribution, which has been found to yield accurate cross sections for $E>2 \mathrm{keV} / \mathrm{amu}$ in collisions with $\mathrm{H}(n=2)$. For the sake of conciseness, we only report in fig. 6 ionization and partial- $n$-CX cross sections. The most prominent feature of this figure is the spreading of the capture flux onto a broad range of $\mathrm{B}^{4+}$ shells; this is a consequence of the initial states being degenerate with the high-lying $\mathrm{B}^{4+}(n=15)$ level, and their energies lie very close to a broad range of $n$-shells of $\mathrm{B}^{4+}$. Accordingly, semiclassical closecoupling calculations would require a prohibitively large number of basis orbitals. Since $n l$-CX partial cross sections are needed to evaluate the effective emission coefficients, they have been also computed in the CTMC calculation, and they are available from the authors upon request. 


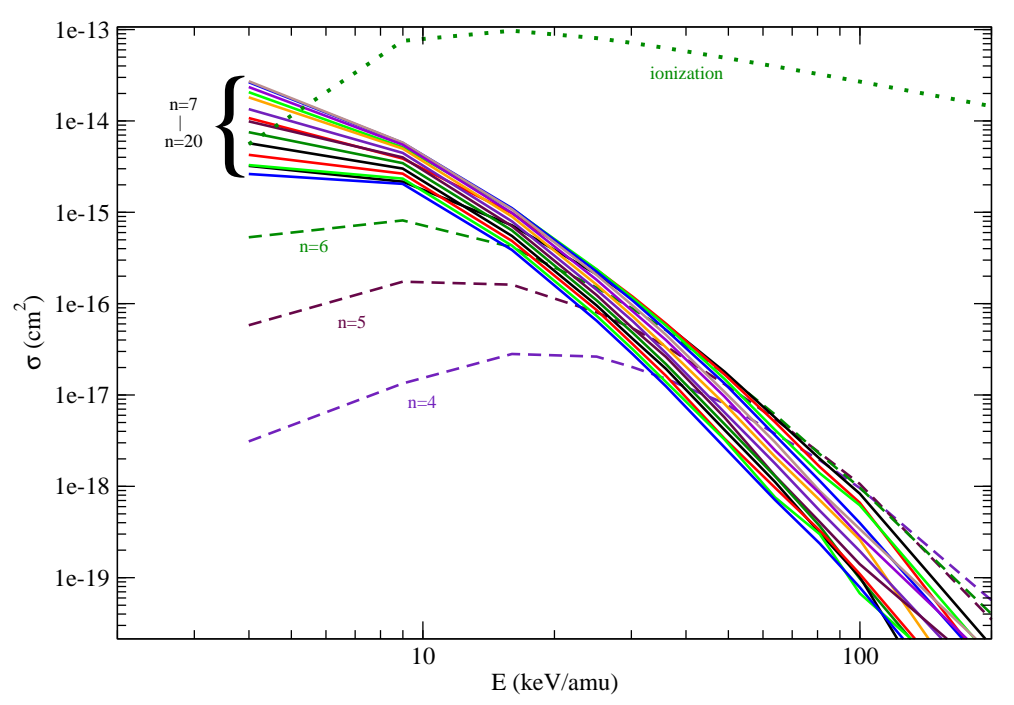

Figure 6. Total cross sections for $\mathrm{CX}$ into $\mathrm{B}^{4+}(n)$ and ionization in collisions of $\mathrm{B}^{5+}$ with $\mathrm{H}(n=3)$ evaluated using the CTMC method with a microcanonical initial distribution.

\subsection{Effective emission coefficients}

The integrated photon emissivity in the line of sight given by a transition decay in an impurity of nuclear charge $\mathrm{Z}$ after a CX collision is given by:

$$
I_{n n^{\prime}}^{Z-1}=\int q_{n \rightarrow n^{\prime}}^{n_{i}} N_{\mathrm{H}}\left(n_{i}\right) N_{Z} \mathrm{~d} s
$$

where $N_{\mathrm{H}}\left(n_{i}\right)$ is the number density of the donor (Hydrogen) in the energy level $n=n_{i}$ and $N_{Z}$ the number density of the ionized impurity (B ${ }^{5+}$ in this work). $q_{n \rightarrow n^{\prime}}^{n_{i}}$ are the effective emission coefficients; they contain the contributions of all the spontaneous $n l$-sublevels transitions and the cascade corrections from the CX populated levels that decay into the $n$ level, calculated by means of a Generalized Collisional Radiative model $[19,21]$.

In order to discuss the relevance of our data in real practical conditions, and to compare with those already present in the ADAS database, we have used the ADAS implementation [19] to calculate ASDEX relevant effective coefficients; we have taken the plasma conditions of ASDEX discharges n. 19364-65 [20]. We have used the electronic $\left(T_{e}\right)$ and ionic $\left(T_{i}\right)$ temperatures $T_{e}=T_{i}=3 \mathrm{keV}$; electronic density, $N_{e}=5 \times 10^{13} \mathrm{~cm}^{-3}$, and plasma ions density, $N_{i}=4 \times 10^{13} \mathrm{~cm}^{-3}$, with $Z_{\text {eff }}=1.7$. The magnitude of the magnetic field is $3 \mathrm{~T}$. The emission coefficients for the $7 \rightarrow 6$ transition are shown in figure 7. There is a significant difference between the coefficients $q_{7 \rightarrow 6}^{1}$ at $E<80 \mathrm{keV} / \mathrm{amu}$ which is due to the differences found in [5] between the corresponding cross sections; those stored in ADAS were obtained by means of an interpolation using results from atomic-orbital-close-coupling (AOCC) calculations of ref. [22] for $E \leq 50 \mathrm{keV} / \mathrm{amu}$ and unitarized-distorted-wave (UDW) calculations of ref. [23] for $E>50 \mathrm{keV} / \mathrm{amu}$. Besides the deviations caused by the interpolation, the discrepancies between the ADAS and 
present cross sections can be related to the difficulties that face AOCC calculations in providing accurate cross sections for populating high-lying capture channels at low $E$. These channels are populated at small internuclear distances through rotational and radial transitions from lowest channels, and at large $R$ 's through a serie of adjacent (molecular) pseudocrossings (see Fig. 2). The AOCC method can be faced with difficulties in describing the molecularization of the electron cloud which comes into play in both small- and large- $R$ mechanisms [24]. Reversely, two-center effects are well represented in both MOCC and CTMC frameworks which are presently used to build our recommended cross sections and calculate the emission coefficients. For $E>80$ $\mathrm{keV} / \mathrm{amu}$, our computed and ADAS coefficents suitably coalesce since improved CTMC and perturbative methods such as UDW are known to yield similar CX cross sections (see, for instance, [25]).

Our effective emission coefficients $q_{7 \rightarrow 6}^{2}$ are similar to those computed from ADAS data. Such an agreement was to be expected since the underlying cross sections stored in ADAS are issued from microcanonical CTMC calculations [6] which, as above mentioned, provide CX cross sections in good agreement with the present calculations (see Fig. 5).

The values $q_{7 \rightarrow 6}^{3}$, displayed in Fig. 7 as function of the impact energy $E$, are of the same order of magnitude than $q_{7 \rightarrow 6}^{2}$. This indicates that the relevance of $\mathrm{H}(n=3)$ in the CXRS diagnostic will be relatively small, unless a very high beam excitation takes place.

\section{Summary}

We have evaluated $\mathrm{CX}$ cross sections in $\mathrm{B}^{5+}+\mathrm{H}\left(n_{i}=2\right)$ collisions using a close-coupling semiclassical molecular treatment and an eikonal-CTMC treatment with an improved initial distribution. In contrast with previous calculations, our results agree with the values obtained using a microcanonical distribution and with the CTMC values available in the ADAS database. This agreement is due to the fact that CX from excited H mainly occurs by means of overbarrier transitions whose description does not require the use of an improved initial CTMC state.

The effective coefficients for the $\mathrm{B}^{4+} n=7 \rightarrow n=6$ emission after $\mathrm{B}^{5+}+\mathrm{H}(1 s)$ CX show a significant deviation with respect to the ADAS values for $E<50 \mathrm{keV} / \mathrm{amu}$. We traced back the root of this discrepancy to the inadequacy of the underlying cross sections stored in ADAS.

We have also evaluated CX cross sections in $\mathrm{B}^{5+}+\mathrm{H}\left(n_{i}=3\right)$ using the eikonalCTMC treatment. The ensuing effective emission coefficient for ASDEX-U plasma parameters is of the same order of magnitude than the corresponding one for CX collisions with $\mathrm{H}\left(n_{i}=2\right)$ donor. We conclude that $\mathrm{CX}$ from $\mathrm{H}\left(n_{i}=3\right)$ could be relevant in high-density conditions, as those of the Alcator C-Mod tokamak [8]. 


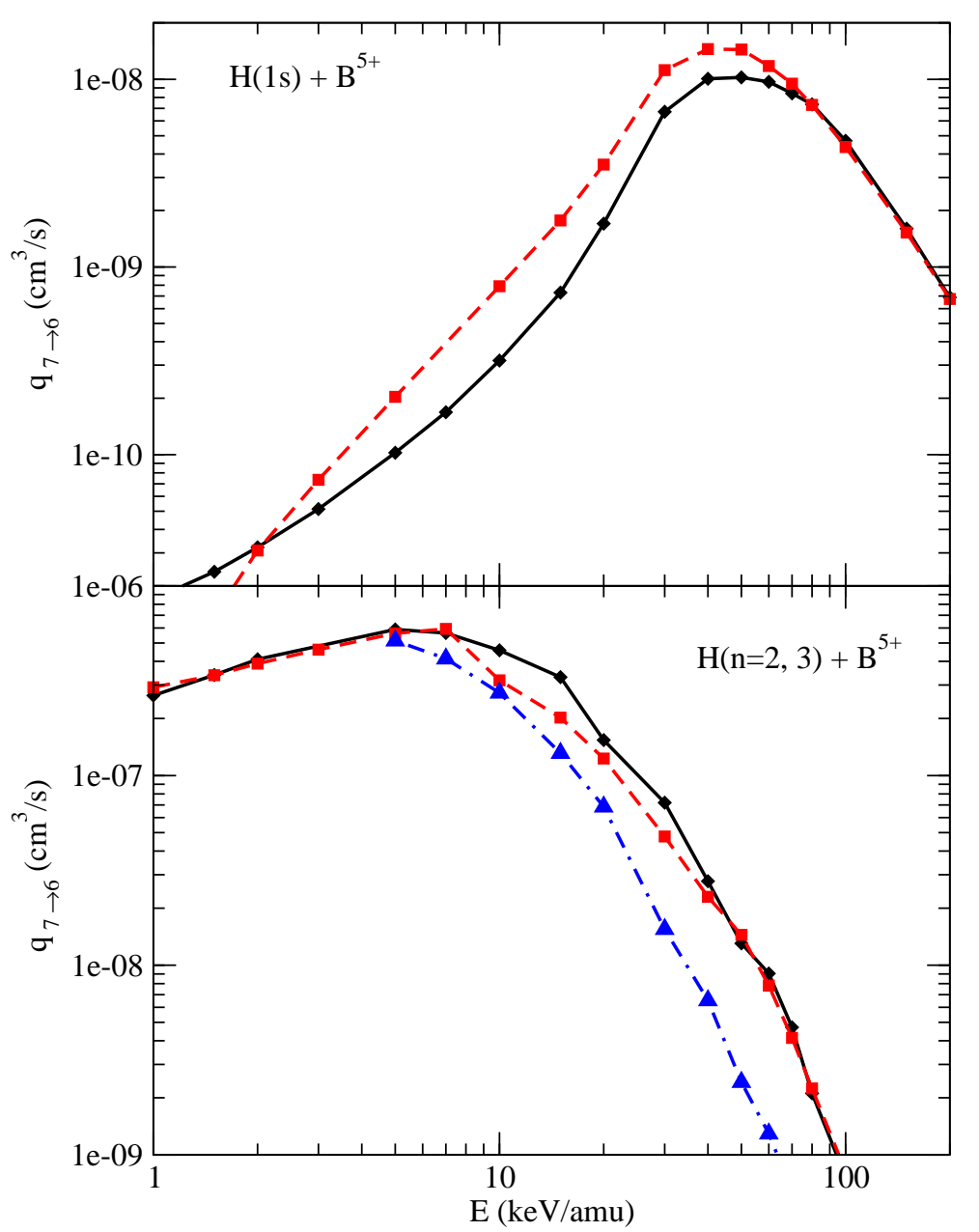

Figure 7. Effective emission coefficients $q_{7 \rightarrow 6}^{n_{i}}$ with $n_{i}=1-2$ (dashed lines with squares), compared with those evaluated using the CX cross sections stored in ADAS (full lines). The line with triangles are the calculated values of $q_{7 \rightarrow 6}^{3}$

\section{Acknowledgments}

This work has been supported by the project ENE2007-62934 of the Ministerio de Ciencia e Innovación (Spain).

\section{References}

[1] O. Marchuk, G. Bertschinger, W. Biel, E. Delabie, M. G. von Hellermann, R. Jaspers, and D. Reiter. Review of Scientific Instruments, 79(10):10F532, 2008.

[2] R. C. Isler. Plasma Phys. Control. Fusion, 36:171, 1994.

[3] L. F. Errea, C. Illescas, L. Méndez, B. Pons, A. Riera, and J. Suárez. J. Phys. B: At. Mol. Opt. Phys., 37:4323, 2004.

[4] L F Errea, Clara Illescas, L Mendez, B Pons, A Riera, and J Suarez. J. Phys. B: At., Mol. Opt. Phys, 39(5):L91, 2006.

[5] L F Errea, F Guzmán, Clara Illescas, L Méndez, B Pons, A Riera, and J Suárez. Plasma Phys. Control. Fusion, 48(11):1585-1604, 2006. 
[6] R Hoekstra, H Anderson, F W Bliek, M von Hellermann, C F Maggi, R E Olson, and H P Summers. Plasma Phys. Control. Fusion, 40(8):1541, 1998.

[7] C. F. Maggi, L. D. Horton, B. Zaniol, P. Franzen, H. Meister, D. Nishijima, and A. Stäbler. In 30th EPS Conference on Controlled Fusion and Plasma Physics, volume 27A, page 1.63. Europhysics Conference Abstracts, 2003.

[8] I. O. Bespamyatnov, W. L. Rowan, R. S. Granetz, and D. F. Beals. volume 77, page 10F123. AIP, 2006.

[9] F. L. Tabarés, D. Tafalla, R. Balbín, B. Brañas, T. Estrada, I. García-Cortés, F. Medina, and M.A. Ochando. Journal of Nucl. Mat., 313-316:839, 2003.

[10] L F Errea, F Guzman, Clara Illescas, L Mendez, B Pons, A Riera, and J Suarez. Journal of Physics: Conference Series, 58:203, 2007.

[11] L. F. Errea, C. Harel, H. Jouin, L. Méndez, B. Pons, and A. Riera. J. Phys. B: At. Mol. Opt. Phys., 31:3527, 1998.

[12] R. Abrines and I. C. Percival. Proc. Phys. Soc., 88:861, 1966.

[13] D. J. W. Hardie and R. E. Olson. J. Phys. B: At. Mol. Phys., 16:1983, 1983.

[14] L. F. Errea, C. Illescas, L. Méndez, B. Pons, A. Riera, and J. Suárez. Phys. Rev. A, 70:52713, 2004.

[15] R. L. Becker and A. D. MacKellar. J. Phys. B: At. Mol. Phys., 17:3923, 1984.

[16] S. B. Schneiderman and A. Russek. Phys. Rev., 181:311, 1969.

[17] C. Harel and H Jouin. J. Phys. B: At. Mol. Opt. Phys., 24:3219, 1990.

[18] A. Salin. J. Phys (Paris), 45:671, 1984.

[19] H. P. Summers. The ADAS User Manual 2.6, 2006.

[20] C. F. Maggi and F. Guzmán. Private Commuinication, 2009.

[21] H P Summers, W J Dickson, M G O'Mullane, N R Badnell, A D Whiteford, D H Brooks, J Lang, S D Loch, and D C Griffin. Plasma Phys. Control. Fus., 48(2):263, 2006.

[22] W. Fritsch and C. D. Lin. Phys. Rev. A, 29:3039, 1984.

[23] H. Ryufuku. Technical Report JAERI-M-82-031, Japan Atomic Energy Research Institute, Tokai, Ibaraki, Japan, 1982.

[24] W. Fritsch and C. D. Lin. J. Phys. B: At. Mol. Phys., 15:1255, 1982

[25] C. Illescas and A. Riera. Phys. Rev. A, 60:4546, 1999. 